\title{
Introduction to Online Learning Volume 23, Issue 2
}

\author{
Peter Shea \\ University at Albany, SUNY \\ Editor-in-Chief of Online Learning
}

Our second issue of Online Learning for 2019 brings readers a diverse collection of papers on topics related to faculty development, student issues, pedagogy, tools, and support. This edition concludes with a book review.

The first paper is "The Effectiveness of Professional Development in Overcoming Obstacles to Effective Online Instruction in a College of Education" by Jered Borup and Anna Evmenova of George Mason University. The growth of online learning has led to greater demand for better prepared instructors at all levels of education. This may be most important at the university level in schools of education that are increasingly tasked with preparing online teachers at the K-12 level. The authors of this study investigate a program designed to improve faculty members' knowledge, skills, and dispositions for teaching online courses. Using a qualitative case study design, they analyzed interview, survey, and posting data of 18 faculty in the professional development course. They conclude that modeling strong pedagogy in the online course had the greatest impact on participants.

The next study in the current issue begins a section on lessons from the field. These papers reflect systematic case study efforts to gain insight into approaches that improve practice. The title of the initial paper is "Putting Theory Into Practice: Incorporating a Community Engagement Model Into Online Pre-Professional Courses in Legal Studies and Human Resources Management" by Antoinette France-Harris, Christine Burton, and Mara Mooney of Clayton State University. The authors used community engagement projects, a pedagogical tool that combines classroom learning goals with targeted community service, to understand whether these projects create an environment that supports forms of productive interaction and generally enhance course quality. They found that while students learn the basics of their profession in the classroom, the human side can be difficult to replicate; but this deficiency was resolved in the community engagement projects.

The next paper is "Promoting a Sense of Belonging in Online Learning Communities of Inquiry in Accredited Courses" by Susi Peacock of Queen Margaret University, Scotland, (now at the University of the West of England) and John Cowan of Edinburgh Napier University, Scotland. The authors focus on the topic of creating a sense of learner belonging in online education through a discussion of a revised version of Community of Inquiry (CoI) framework. This revision of the well-known framework emphasizes the overlapping intersections of the three presences, defining these overlaps as trusting, meaning-making, and deepening understandings. Suggestions are provided for each of these concepts, leading to particular examples that highlight the advancement of a sense of belonging as an important aspect of the online instructor's teaching activities.

The third paper in this section is "Open Video Repositories for College Instruction: A Guide to the Social Sciences" by Michael Miller of University of Texas at San Antonio and A. S. CohenMiller of Nazarbayev University, Kazakhstan. This paper documents the quantity and 
quality of open educational resources, specifically video for instructional materials, in the social sciences. Video content for instruction on these sites ranges from economics, psychology, political science, sociology, anthropology, and history. Some multidisciplinary sites exist as well. This guide will prove useful for faculty and instructional designers looking to enhance their instruction with free or low-cost instructional resources.

The next section of the journal investigates a set of common themes around students, pedagogy, tools, and support. The first paper, which might be a companion to the previous paper, is "Designing and Developing Videos for Online Learning: A Seven-Principle Model" by Chaohua $\mathrm{Ou}$, David Joyner, and Ashok Goel of the Georgia Institute of Technology. In this study the authors used seven principles from instructional design theories to guide the design and development of video lessons for an online graduate course. The authors integrated instructional presentation with instructional methods and sequencing. They assessed the effectiveness of this model through student surveys for eight semesters. This paper presents findings as well as the instructors' experience of designing and developing the video lessons. Implications of the findings for instructional design and future research are also discussed.

The next paper is "Student Engagement as Predictor of xMOOC Completion: An Analysis From Five Courses on Energy Sustainability" by Brenda Edith Guajardo Leal and Jaime Ricardo Valenzuela González of Tecnologico de Monterrey, Mexico, and John Scott, University of California, Berkeley. This study seeks to understand causes of dropout in Massive Open Online Courses (MOOCs). The authors used two samples - one of all participants and one of a sample of participants who completed a survey. Results suggest that, overall, the more frequent the individual's participation in the forum and the higher their level of educational attainment, the more likely it was that the participant would complete the course. It is difficult to determine the direction of causality - were those participants who were more engaged more likely to contribute to the forum, or did forum participation increase engagement and therefore persistence in the course? Other consistent findings were that older participants were more likely to complete the course (though the age difference between completers and noncompleters was very small). This study thus raises as many questions as it answers, which may be useful for future researchers.

The seventh paper in this issue is "The Efficacy of an Online Cognitive Assessment Tool for Enhancing and Improving Student Academic Outcomes" by Lindsay Shaw, Janet MacIsaac, and Jill Singleton-Jackson of the University of Windsor, Canada. In this paper the authors used an online application to compare students' level of engagement and test performance to their final, multiple-choice classroom-based exam grade to assess the effectiveness of the application in promoting improved learning outcomes. The study controlled for a variety of other factors, such as learning orientation, grade orientation, and test anxiety. The results suggest that learners performed well on the online assessment despite their limited engagement in the cognitive learning features of the application. It appears that students did not utilize the built-in cognitive learning strategies embedded in the application and chose instead to move directly to the multiple-choice assessments, which were graded. The results highlight the challenges associated with engaging students with formative online assessment environments, and they may prove useful to future researchers who seek to improve on these results.

The next paper is "Learner Engagement in Blended Learning Environments: A Conceptual Framework" by Lisa Halverson and Charles Graham of Brigham Young University. The study reviews the literature related to harnessing learners' cognitive and emotional resources to accomplish learning tasks to create a model that describes and explains learner engagement when 
some educational activity is carried out in classrooms and some occurs online. This review of the literature helps us overcome the imprecise ways in which engagement is discussed, provides specific guidance for further research, and focuses on an increasingly relevant context in higher education - blended learning.

The ninth study in this issue is "Posting Patterns of Students' Social Presence, Cognitive Presence, and Teaching Presence in Online Learning" by Selcan Kilis of Giresun University and Zahide Yıldırım of Middle East Technical University, Turkey. This case study examines asynchronous online discussions using the well-known indicators of teaching, social, and cognitive presence described in the CoI framework. The authors make a case that students exhibit higher levels of social and teaching presence when discussions are structured to elicit them. Of particular concern, past research has indicated that students do not reach high levels of cognitive presence through online discussion activities, which are common in online education. In this study, the topics for discussion were chosen based on real-life situations to motivate brainstorming and critical thinking and to increase the use of students' life experiences. The authors suggest that this purposeful design holds promise in developing more productive communities of inquiry in online settings.

The next study is "A Structural Equation Model of Predictors of Online Learners' Engagement and Satisfaction" by Sevda Kucuk of Istanbul University-Cerrahpasa and Jennifer Richardson of Purdue University. This study presents a different research perspective on the CoI framework than the previous paper, employing alternative analytic approaches with a larger sample of students and correlating the indicators with measures of satisfaction. The authors also investigate other measures of engagement, including emotional, behavioral, cognitive, and agentive indicators, and they develop hypotheses correlating these with $\mathrm{CoI}$ indicators to predict the outcome measure of satisfaction and explore relationships between CoI and engagement indicators. The paper uses structural equation modeling of survey results to develop a predictive model. Regarding the predictors of engagement, the authors conclude that cognitive presence is the primary predictor of emotional, cognitive, and behavioral engagement and that it had an indirect effect on agentic engagement. The path analysis further indicates that the prevailing determinant of satisfaction was teaching presence, which showed direct and indirect effects on satisfaction. They conclude that the model could serve as a foundation for pedagogical improvement of both satisfaction and engagement in online learning environments.

The next paper is "Scribe Hero: An Online Teaching and Learning Approach for the Development of Writing Skills in the Undergraduate Classroom" by Kimberly Francis, Jodie Salter, Lucia Costanzo, and Serge Desmarais of the University of Guelph; Meagan Troop of Sheridan College; and Rosheeka Parahoo of Western University, Canada. This mixed-methods paper describes and assesses an online environment created to help students learn academic writing skills (planning, writing, citation, and grammar) and designed to reinforce what previous research has identified as three key features of motivating online experiences: interactivity, agency, and engagement. Quantitative results of the study suggest that students' acquisition of writing skills was enhanced following their interaction with the online learning modules developed for the study. For those students who completed all four modules, there was consistent improvement in posttest scores. When used with full functionality, including feedback and badges, the intervention was even more effective. Qualitative results indicate that many students appreciated and enjoyed the flexibility and format of online learning as a means for developing writing skills. This study will 
be useful for other researchers and practitioners seeking to investigate online instruction in the service of improving academic writing.

The final paper in this issue is a review of the book Blended Learning in Action: A Practical Guide Toward Sustainable Change. The authors of the book are Catlin Tucker, Tiffany Wycoff, and Jason Green. The review is by Heidi Rowland of Boise State University. This book is a guide for integrating blended learning into K-12 environments, and the review provides excellent insights that will assist those interested in learning more (including many in higher education).

We invite you to read, share, and cite articles in this issue and to consider submitting your own original work to Online Learning. 